\title{
Reconstruction and dynamics of human intestinal microbiome observed in situ
}

2 Xiaolin $\mathrm{Liu}^{1,2^{*}}$, Min $\mathrm{Dai}^{3 *}$, Yue $\mathrm{Ma}^{1,2}, \mathrm{Na} \mathrm{Zhao}^{1}$, Huijie Zhang ${ }^{4}$, Liyuan Xiang ${ }^{3}, \mathrm{He}$

3 Tian ${ }^{5}$, Guanghou Shui ${ }^{5}$, Faming Zhang ${ }^{3,6 \#}$, Jun Wang ${ }^{1,2 \#}$

5 1. CAS Key Laboratory of Pathogenic Microbiology and Immunology, Institute of

6 Microbiology, Chinese Academy of Sciences, Beijing 100101, China.

7 2. University of Chinese Academy of Sciences, Beijing 100049, China.

8 3. Medical Center for Digestive Diseases, the Second Affiliated Hospital of Nanjing

$9 \quad$ Medical University, Nanjing 210011, China.

10 4. Clinical Trial Center Office, the Second Affiliated Hospital of Nanjing Medical 11 University, Nanjing 210011, China.

5. State Key Laboratory of Molecular Developmental Biology, Institute of Genetics and Developmental Biology, Chinese Academy of Sciences, Beijing, 100101,

14 China.

6. Key Lab of Holistic Integrative Enterology, Nanjing Medical University, Nanjing 210011, China.

7.

18 Xiaolin Liu and Min Dai contributed equally to this study. 
27 Prof. Jun Wang, CAS Key Laboratory of Pathogenic Microbiology and Immunology,

28 Institute of Microbiology, Chinese Academy of Sciences, No. 1-3 Beichenxi Road,

29 Chaoyang District, Beijing 100049, China.

30 E-mail: junwang@im.ac.cn

32 Prof. Faming Zhang, Medical Center for Digestive Diseases, the Second Affiliated

33 Hospital of Nanjing Medical University, 121 Jiang Jia Yuan, Nanjing 210011, China.

Fax: $+86-025-58509931$

Telephone: +86-025-58509883 


\section{Abstract}

Gut microbiome are studied primarily using fecal samples in humans and we gained vital knowledge of compositional and functional capacities of gastro-intestinal microbial communities. Yet, fecal materials limit our ability to investigate microbial dynamics in different locations along GI-tract (in situ), nor in finer temporal scales as they are infrequent. With a technology developed originally for fecal material transplantation, colonic transendoscopic enteral tubing, we were able to sample ileocecal microbiome twice daily, and carried out metagenomic as well as metatranscriptomic analyses. Ileocecal and fecal microbiome are similar in metagenomic profiling, yet their active genes (in metatranscriptomes) are highly distinct. Both were perturbed after laxatives and then became more similar to microbiome prior to treatment, demonstrating resilience as an innate property of gut microbiome. Ileocecal microbiome transcriptomes sampled during day and night revealed diurnal rhythmes exist in certain bacterial species and functional pathways, in particular those related to short-chain fatty acid production. Lastly, metabolomic analysis in fecal and urine samples mirrored the perturbance and recovery in gut microbiome, indicating crucial contribution of gut microbiome to many of the key metabolites involved in host health. Our study provides interesting novel insights into human gut microbiome, and demonstrates the inner resilience, diurnal rhythmes and potential consequences to the host. 


\section{Introduction}

68 Investigations into human gut microbiome has revealed the pivotal role of microbial

69 communities in host health and disease ${ }^{1}$, however the majority of the studied

70 microbiome are from defecated feces, which are powerful representations of

71 gastrointestinal (GI) microbial ecosystems yet still with limitations. Fecal microbiome

72 largely represent the last stage of transitions of microbial communities along the GI

73 tract, and as numerous biogeographical analyses revealed the distinction of fecal

74 microbiome from different GI sections ${ }^{2}$, where important biological processes take

75 place. Largely composed of bacteria and archaea from lumen (content), fecal

76 microbiome also indicate poorly on the mucosal microbiome, community at the

77 intimate interface of cross-talk with the host and especially interactions with immune

78 systems $^{3,4}$. In addition, continual fecal samples are rarely taken within short intervals

79 but mostly daily due to human physiology, which disables investigations into

80 community dynamics with finer resolution, for instance diurnal cycles that are feasible

81 in mouse and have already been shown to be closely linked to host physiological

82 homeostasis $5,6,7$.

83 While most of the time-series studies of human gut microbiome avoid intentional

84 challenges or stress, perturbations in human gut microbiome are common and impact

85 their composition and functions ${ }^{8,9}$, and the shifts as well as dynamics are commonly

86 indicative of community robustness under environmental stress. Extreme dietary

87 changes are linked to quick responses of human gut microbiome in healthy individuals ${ }^{8}$,

$89^{9}$, while longer-term dietary habits are shown to be associated with more generalized 
89 microbiome clusters known as enterotypes ${ }^{10,11}$. Gut microbiome response distinctively

90 towards many classes of antibiotics ${ }^{12,13}$, and the effects can sometimes detectable after

91 months or years since last administration, though such studies are usually

92 retrospective ${ }^{14,15,16}$. Continuously monitoring of human gut microbiome after infection

93 or medical treatment has achieved interval of days after the event or interventions ${ }^{17}$,

94 and study into one type of large-scale replacement of gut microbiome, fecal microbiota

95 transplantation (FMT), demonstrated that an foreign microbiome can partially establish

96 and replace the original gut microbiome, resulting in a mosaic of original and new gut

97 microbiome ${ }^{18}$. Osmotic laxatives can remove large portions of GI microbiome and

98 widely used for treating constipation but also before colonoscopy ${ }^{19}$, 20 , which is linked

99 with long-term partially alterations of gut microbiota after a week of continuous

100 administration in mice, with negative consequences on host intestinal and

101 immunological homeostasis 5 .

102 Feces usually recover to previous physical state within days after osmotic laxatives

103 in healthy individuals, as observed in clinical practices. Such reconstruction process

104 provides a fascinating opportunity to examine the dynamics and robustness of healthy

105 human gut microbiome ${ }^{21,22}$, after strong perturbation in terms of quantitative change in

106 microbiome. In addition, a technique of placing a tube through the anus into cecum or

107 terminal ileum under endoscopy for repeated administration of FMTs or medications is

108 called colonic transendoscopic enteral tubing (TET) ${ }^{23,24}$. The TET tube also reversely

109 allows sampling of fluid in intestine, facilitates analyses of both fecal microbiome after

110 osmotic laxatives and also ileocecal microbiome in situ. In invited healthy volunteers 
111 adminisrating osmatic laxatives, the dynamics of reconstruction in fecal microbiome

112 on daily basis, and ileocecal microbiome with a 12-hour interval to investigate fine-

113 resolution dynamics that might contribute to host circadian rhythms were investigated,

114 empowered by combined metagenomic, metatranscriptomic and metabolomic analyses.

\section{Results}

116 Five healthy volunteers underwent osmotic laxatives treatment, colonoscopy and

117 colonic TET in our study. Fecal samples were collected before taking laxatives as well

118 as all days remaining TET (Figure 1). Ileocecal fluid were sampled each day at 10 am

119 as well as $10 \mathrm{pm}$, washed off using normal saline solution and collected through TET

120 using syringe. In total, metagenomic DNA as well as RNA were extracted from 28 fecal

121 and 43 ileocecal samples for metagenomic and metatranscriptomic sequencing and

122 analysis ${ }^{25,26}$ on Illumina NovaSeq platform. Urine samples were also collected from

123 each volunteer and together with fecal samples from the same sampling time, were

124 analyzed for metabolome.

125 Fecal and ileocecal microbiome are distinct in composition and functionality

126 Analysis of standing (DNA-based) and active (RNA-based) microbial communities

127 revealed their distinct features both in defecated feces samples as well as in ileocecal

128 samples. For fecal samples collected before osmotic laxatives, standing communities

129 are largely dominated by species Alistipes putredinis, Bacteroides stercoris, Prevotella

130 stercorea, Faecalibacterium prausnitzii, Bacteroides massiliensis among others

131 (Figure 1, Figure S1). Yet those microbial species do not always occupy the majority

132 in RNA reads, rather, species Ruminococcus torques, Methanobrevibacter, 
133 Subdoligranulum, Faecalibacterium prausnitzii, Ruminococcus obeum are more

134 prominent in RNA-based surveys (Figure 1, Figure S1). This could be extended for

135 functional pathway analysis, for the most abundant pathways in metagenomic DNA are

136 adenosine ribonucleotides de novo biosynthesis, UMP biosynthesis, UDP-N-

137 acetylmuramoyl-pentapeptide biosynthesis II (lysine-containing), UDP-N-

138 acetylmuramoyl-pentapeptide biosynthesis I (meso-diaminopimelate containing) and

139 peptidoglycan biosynthesis I (meso-diaminopimelate containing) (Figure 1, Figure

140 S2). While adenosine ribonucleotides de novo biosynthesis remains the highest in

141 abundance in metatranscriptome, glycolysis IV \& III, starch degradation V and

142 gluconeogenesis I are actually among the highest in RNA reads (Figure 1, Figure S2).

143 Similar distinction could be observed in ileocecal samples as well as fecal samples

144 collected post-colonoscopy, that DNA-based profiling of microbial composition or

145 functionality, even though most widely used, does not represent the actually status of

146 the microbial communities in terms of activity. Many of the abundant taxa in DNA-

147 based profiling were found to be with low activity in terms of RNA-transcription, while

148 many metabolic pathways found highly expressed in metatranscriptomic analysis were

149 very low in metagenomic (DNA) reads (Figure 1). The reverse was also true for many

150 bacterial species as well as metabolic pathways.

151 Ileocecal microbiome, collected in situ, reveal essential differences in taxonomy

152 and functional pathways when compared to fecal samples mainly at actively transcribed

153 genes (metatranscriptome). At DNA level, species composion and pathways are very

154 similar between ileocecal and fecal samples from anus, without significant differences. 
155 Yet at transcriptome level, fecal and ileocecal samples showed essential differences,

156 Collinsella unclassified, Bifidobacterium longum, Bifidobacterium adolescentis,

157 Ruminococcus obeum are highly active in ileocecal microbiome while Parabacteroides,

158 Subdoligranulum, Faecalibacterium prausnitzii, Bacteroides uniformis, Bacteroides

159 vulgatus are in fecal materials (Figure 2, Figure S3). In terms of functional pathways,

160 adenosine ribonucleotides de novo biosynthesis, glycolysis IV \& III and guanosine

161 ribonucleotides de novo biosynthesis are enriched in fecal microbiome RNA,

162 suggesting high metabolic activities, while gluconeogenesis I, superpathway of

163 beta-D-glucuronide and $\mathrm{D}-$ glucuronate degradation, superpathway of sulfur amino

164 acid biosynthesis, tRNA processing and dTDP-N-acetylthomosamine biosynthesis are

165 potentially most active in ilealcecal samples.

166 Fecal microbiome dynamically reconstruct after osmotic laxatives treatment

167 With strong perturbation of osmotic laxatives, fecal microbiome of each volunteer all

168 showed dynamic recovery/reconstruction in the days following colonoscopy. Earliest

169 fecal samples after laxatives showed differences in bacterial composition, both at DNA

170 and RNA level. In DNA-based analysis, the most dominant taxa in the earliest fecal

171 samples post-laxatives are Bacteroides plebeius, Bacteroides massiliensis, Bacteroides

172 stercoris, Bacteroides vulgatus and Megamonas unclassified, instead of Alistipes

173 putredinis, Bacteroides stercoris, Prevotella stercorea, Faecalibacterium prausnitzii

174 and Bacteroides massiliensis in the fecal samples before (Figure S4). This points to

175 either different sensitivity to laxatives among different species in the process of being

176 washed out, or variations in growth/recovery within the short period of laxatives to next 
177 episode of defecation. Active members, suggested by RNA analysis, also showed that

178 the most active species in fecal samples changed from Ruminococcus torques,

179 Methanobrevibacter unclassified, Subdoligranulum unclassified, Faecalibacterium

180 prausnitzii and Ruminococcus obeum before laxatives to Parabacteroides unclassified,

181 Ruminococcus torques, Collinsella unclassified, Collinsella aerofaciens and Dorea

182 longicatena immediately post-laxatives (Figure S4). Laxatives also caused shifts in

183 abundances of metabolic pathways in DNA and RNA based analysis. The most

184 abundant pathways changed to adenosine ribonucleotides de novo biosynthesis, UDP-

185 N-acetylmuramoyl-pentapeptide biosynthesis II (lysine-containing), queuosine

186 biosynthesis, guanosine ribonucleotides de novo biosynthesis and S-adenosyl-L-

187 methionine cycle I in DNA and adenosine ribonucleotides de novo biosynthesis,

188 guanosine ribonucleotides de novo biosynthesis, glycolysis IV \& III and starch

189 degradation V in RNA, instantly after laxatives (Figure 3, Figure S4). In the following

190 days, fecal metagenomics and transcriptomes showed trends of recovery in four out of

191 five volunteers, as demonstrated by the dissimilarities of metagenomic as well as

192 transcriptomic tend to decrease with time, and both principle coordinate analysis (PCoA)

193 as well as compositional analysis could show that after drifting further from the pre-

194 laxative fecal microbiome at certain time points, post-laxative fecal microbiome tend

195 to become more similar to microbiome before osmotic laxative treatment (Figure 3,

196 Figure S5). Interactions between members of microbiome, as well as host

197 physiological factors could both contribute to the recovery of microbiome after

198 perturbation, and confer the individualized resilience of microbial communities. 
As ileocecal microbiome could not be analyzed without colon cleaning and the remained TET tube, it might be impossible to measure the recovery of microbiome

201 using ileocecal content samples, yet their dynamics were also prominent and because

202 of sampling intervals, could be studied with finer temporal resolution than fecal samples.

203 Ileocecal contents, even though distinct in composition and functionality from fecal 204 samples from the same individual and from the same day, mirror the dynamics of fecal 205 microbiome to a large extent and could indicate that the ileocecal microbiome shifts 206 eventually contribute to the dynamics observed in fecal samples (Figure 4). Major 207 species determined by DNA and RNA analysis, as well as dominant metabolic 208 pathways showed similar changes in ileocecal samples and defecated fecal samples, for

209 instance in terms of microbial taxonomy, Bacteroides caccae, Bacteroides uniformis,

210 Coprobacter fastidiosus and Parabacteroides merdae in metagenomic reads,

211 Subdoligranulum in metatranscriptomic reads (Figure 4), and superpathway of 212 histidine (purine) and pyrimidine biosynthesis, superpathway of pyrimidine 213 ribonucleosides degradation, superpathway of UDP-glucose derived $\mathrm{O}$ antigen building 214 blocks biosynthesis, TCA cycle I and pyruvate fermentation to propanoate I pathways 215 in metagenomic reads, and L-isoleucine biosynthesis II, starch degradation $\mathrm{V}$, 216 glycolysis III, glutaryl-CoA degradation and purine ribonucleosides degradation 217 pathways in metatranscriptomic reads (Figure 4). The relative dissimilarity between 218 ileocecal and defecated feces samples before laxatives of each volunteer showed trends 219 of decrease in three out of five individuals in at least one of the 220 metagenomic/metatranscriptomic measures, mirroring that of the fecal samples 
221 collected post-laxatives (Figure 4, Figure S6). It could be assumed that the ileocecal

222 microbiome were also recovering to a similar taxonomical/functional composition to

223 those before laxative treatments, thus demonstrating its own resilience towards 224 perturbations $^{21}$.

225 Bacterial composition and functional pathways show diurnal rhythms in cecum

226 The feasibility of collecting human ileocecal microbiome samples with 12-hour interval

227 provides a unique opportunity to investigate the diurnal rhythms of GI microbiome, and

228 more importantly in situ. With samples from ileocecal collected at 10 am. and $10 \mathrm{pm}$.

229 each day through TET tube, we were able to investigate the dynamics of species

230 composition and metabolic pathways at both DNA and RNA levels. First, based on

231 taxonomical estimation of different bacterial species in metagenomic and

232 metatranscriptomic analysis, it is surprising that the overall community structure has

233 detectable differences between samples collected during the day and those collected in

234 the evening (Figure 4, Figure S7). Furthermore, we observed different species showing

235 regular oscillations in terms of DNA abundances in each volunteer, among which a few

236 were shared by two or more individuals: Streptococcus parasanguinis, Dorea

237 longicatena, Propionibacterium acnes, Ruminococcus lactaris, Lachnospiraceae

238 bacterium_5_1_63FAA and Haemophilus haemolyticus. While DNA-based abundance

239 estimation reveals cell number shifts of different taxa, RNA-based analysis on the other

240 hand demonstrates that many species have diurnal rhythm in overall gene expression

241 (or transcription activities), namely Coprococcus comes, Bifidobacterium

242 pseudocatenulatum, Ruminococcus lactaris, Ruminococcus gnavus, Ruminococcus 
torques, Subdoligranulum and Mitsuokella, the differences in those bacteria with

244 diurnal rhythm among volunteers indicate a personalized trait of microbiome dynamics

245 in the ileocecal microbiome, as well as different reproduction rate/metabolic activities

246 of various taxonomical groups.

247 We further screened for pathways with prominent diurnal rhythms, primarily within

248 transcriptomic data. This analysis again revealed individualized oscillation patterns of

249 metabolic pathways, revealing four to 41 such pathways in each volunteer (Figure 5).

250 Among those, purine nucleobases degradation I, coenzyme A biosynthesis II, L-lysine

251 biosynthesis I \& III, 5-aminoimidazole ribonucleotide biosynthesis I, sucrose

252 degradation III (sucrose invertase) and superpathway of L-lysine, L-threonine and L-

253 methionine biosynthesis II are shared by two or more volunteers, while the rest are

254 confined to only one individual. In comparison, corresponding metabolic pathways in

255 metagenomic data did not show a significant oscillation pattern, suggesting it is mainly

256 the regulation of transcription in those pathways underlies the diurnal pattern, instead

257 of the increase or decrease in genetic copies in metagenome. A general category of

258 metabolic pathways is related to the production of short-chain fatty acids (SCFAs),

259 including pyruvate fermentation to acetate and lactate II and acetyl-CoA fermentation

260 to butanoate II, as well as acetylene degradation in metatranscriptome data. Those

261 metabolic pathways are generally higher in activity at daytime, while lower at night.

\section{Fecal and urinary metabolome reconstruct with microbiome}

263 To further demonstrate the potential effects of disturbance as well as recovery of gut

264 microbiome on the host, we investigated the fecal and urinary metabolome collected 
before laxatives treatment and along fecal sampling after colonoscopy. Firstly, fecal metabolome demonstrated overall differences after colnoscopy (Figure S8), and

267 significant decrease in abundance due to ostomic laxatives, especially in the proportion 268 of citrulline, N-acetyl-L-methionine, L-glutamic acid, N-acetylglutamic acid, 269 allochenodeoxycholic acid, linoleic acid, oleic acid, 5-hydroxyindoleacetic acid, 270 pantothenic acid, xanthine, oxypurinol, 4-trimethylammoniobutanoic acid and 271 lithocholic acid (VIP value $>1, \mathrm{p}<0.05$, see methods). (Figure 6). Metabolome in 272 fecal samples also reconstruct dynamically after colonoscopy and largely correlated to 273 microbiome recovery, resulting in a relatively similar metabolome compared to fecal 274 samples before the disturbance (Figure S8). Analyzing fecal samples post-colonoscopy 275 revealed many associations between bacterial taxa/metabolic pathways and metabolites, 276 however, to different degrees: we found that the composition of active species 277 (determined by metatranscriptome data) has the strongest association with metabolome 278 shifts, as revealed by mantel test (bray-curtis distance of species composion vs bray279 curtis distance of metabolites, $\mathrm{r}=0.38, \mathrm{p}=0.001$ ), while much lower and non280 significant association is found to between DNA-based bacterial composition and fecal 281 metabolites $(r=0.13, p=0.085)$. In summary, fecal metabolites are also perturbed 282 during ostomic laxatives treatment and reconstruct together with gut microbiome 283 afterwards, which in turn could consequently affect metabolism of the host.

284 Microbiome also contribute to changes of urinary metabolome composition as well 285 as reconstruction, although to a lesser degree than that of fecal metabolome (Figure 286 S8). With depletion of gut microbiome, urinary metabolome did not have so many 
metabolites that have decreased, and the most promiment metabolite only has nearsignificant decrease (7-Methylxanthine, vip $>0, p=0.087)$. In urine samples collected

289 after colonoscopy, metabolome demonstrated lesser yet detectable recovery, mirroring

290 the shifts in gut microbiome (Figure S8).Associations between metabolites and 291 microbial taxa/metabolic pathways could also be established, with niacinamide, 1-3292 dimethyluric acid and 4-trimethylammoniobutanoic acid overlapping between urinary

293 metabolome and fecal metabolome (Figure S9). Here again we observed significant 294 correlation between active bacterial species composition and metabolome, revealed by 295 mantel test $(\mathrm{r}=0.28, \mathrm{p}=0.001)$, which is lower than in fecal metabolome and could be 296 the result of less direct input from microbiome together with higher influences of host 297 metabolic activities. Thus, urinary metabolome is also changed by gut microbiome 298 perturbance and recovery again correlates with microbiome reconstruction.

299 Further enrichment analysis of the metabolites decreased in feces indicated the 300 potential importance of microbiome-derived metabolites in many physiological and 301 pathological conditions. Using MetaboAnalyst ${ }^{27}$ we discovered that changes in those 302 metabolites are significantly associated with many metabolic syndromes including 303 Hartnup disease, Short-Bowel disease, among others; and more intriguingly they are 304 indicated in central neural system disorders including seizures and Schizophrenia, re305 affirming the functioning of gut-brain axis is largely via various microbial metabolites 306 (Figure S10). Enrichment results also include "Metabolites affected by diurnal 307 variations" (10-fold enrichement, $15^{\text {th }}$ in terms of significance value), and since we 308 established that part of those metabolites are largely dependent on the presence of gut 
309 microbiome, diurnal variations in gut microbiome can be assumed to be underlying the

310 diurnal variations in the metabolites themselves.

\section{Discussion:}

312 In the present study, we collected fecal samples as well as in situ ileocecal samples

313 continually after laxatives treatment and colonic TET tube that was originally designed

314 for colonic delivery of fecal microbiota or medications. Combining metagenomic,

315 metatranscriptomic and metabolomic analyses, we profiled the composition, function

316 and dynamics in multiple layers of human gut microbiome, and especially revealed the

317 individuality of reconstruction in microbiome composition and functions, which overall

318 showed shared characteristics of internal resilience of gut microbiome. The feasibility

319 of sampling ileocecal microbiome in situ and at fixed time points that are more frequent

320 than defecated feces, provides an unique insights into the diurnal patterns or circadian

321 rhythms in human gut microbiome for the first time. We managed to conclude that such

322 rhythms occur at whole community level in terms of specific bacterial groups and 323 metabolic pathways.

324 Bacterial species as well as metabolic pathways can have distinct patterns when 325 they are examined at DNA ("standing") level and RNA ("active") levels ${ }^{25,26}$. In 326 defecated fecal samples, a collection of bacterial species including Ruminococcus, 327 Subdoligranulum and Faecalibacterium are highly active in terms of transcription and 328 potentially metabolism, and they belong to the increasingly well-appreciated group of 329 butyrate producers, and butyrate is known to be both metabolically as well as 330 immunologically crucial for the host, especially in the context of several metabolic as 
331 well as auto-immune disorders $28,29,30,31$. Except the top pathway being adenosine 332 ribonucleotides de novo biosynthesis that is related to ATP production and thus energy

333 cycling in both DNA and RNA, metabolic pathways related to nutrients metabolism

334 (for instance glucose metabolism) are highly active in transcriptome data, even though 335 pathways related cellular structures (for instance, components of cell walls) are most 336 abundant in DNAs, suggesting a heterogeneous regulation of transcription in pathways 337 aimed at different parts of microbial physiological activities.

After colon cleaning using laxatives, fecal microbiome first showed dramatic 339 changes in composition and functionality, but in the following time points gradually 340 reduced the distinctions to the microbiome before; considering the strong associations 341 between cecal samples collected with fecal samples at the same time, it could be 342 concluded that the cecal microbiome were dynamically reconstructing as well. Such 343 construction has both the characteristics of high individuality, that different bacterial 344 species/metabolic pathways recover to more similar abundances in each individual; and 345 the shared characteristic of microbial resilience, that they became more and more 346 similar in general to the microbiome pre-laxatives. Many studies have showen that the 347 long-term stability and resilience of gut microbiome after acute disturbance including 348 dietary intervention, medication etc $21,32,33,34$; and microbiome after one-dosage of 349 laxatives is yet another demonstration of such resilience, residing in the internal mutual 350 interactions of microbial species ${ }^{35,36,37}$.

351 Regular, twice daily sampling of ceccal samples provides interesting insights into 352 the diurnal patterns of human GI microbiome, for fecal samples are usually available 
on once per one-two days in humans. In metatranscriptome analysis of cecal samples,

354 butyrate-producing bacteria again compose the majority of species that showed strong

355 signatures of diurnal patterns, potentially a response to food intake and/or host

356 physiological shifts in the GI tract ${ }^{38}$. Metabolic pathways related to production of

357 SCFAs are among those with most prominent diurnal patterns, and those SCFAs have

358 been recognized to be key in affecting host circadian rhythms ${ }^{7,39}$. In addition, recent

359 study has shown that circadian rhythm of host intestinal epithelial cells can be affected

360 by MyD88-dependent HDAC3 gene expression changes, while the activities of HDAC

361 are known to be also affected by SCFAs ${ }^{40,41,42}$. They do not only indicate replication

362 dynamics as well as metabolic activities of bacterial groups during day or night, but

363 might provide the eventual microbial cue for this route of gut microbiota-host cross-

364 talk.

365 Contrary to the previous study in mice ${ }^{5}$, in which laxatives were administrated for

366 a long-time period, volunteers in our study only received once of colon cleaning before

367 colonoscopy and consequently the gut microbiome showed recovery instead of long-

368 term dysbiosis; this agrees with clinical observation that colonoscopies are generally

369 not associated with long term adverse effects in GI systems. Admittedly we did not

370 sampling fecal samples longer enough to judge for whether gut microbiome showed

371 total recovery, as corresponding cecal sampling is no longer feasible after TET tube

372 falls out. The time of remaining colonic TET tube within colon generally ranged one to

373 two weeks if using 2-4 clips for endoscopic fixaton. We are also limited on properly

374 analyzing the ceccal sample metabolome, since the ceccal samples are wash-outs with 
375 undeterminable levels of dilutions, quantification of metabolites became difficult. And

376 at last, host responses can be only inferred by metabolome in the fecal and urine samples,

377 from which we did observe similar-to-microbiome recovery and re-affirmed the

378 contribution of gut microbiome to metabolites, plus the largely microbiome-derived

379 metabolites could be underlying important metabolic and central neural system

380 functions, re-affirming the contribution of gut microbiome in host metabolism and gut-

381 brain axis; and more direct measures including host intestinal epithelial gene

382 expressions are not yet achievable in humans, only in experimental animals.

383 In conclusion, our study investigated the reconstruction of gut microbiome after

384 laxative-caused depletion, and more importantly cecal microbiome with higher

385 temporal resolution than before and in situ. We first time provided direct evidence of

386 diurnal patterns of cecal microbiome in humans, at compositional and functional level.

387 The dynamics of fecal and cecal microbiome, at multi-omics level, facilitates our

388 understanding into gut microbial ecosystem itself, as well as how could they potentially

389 affect host physiological including circadian rhythms.

390 
a

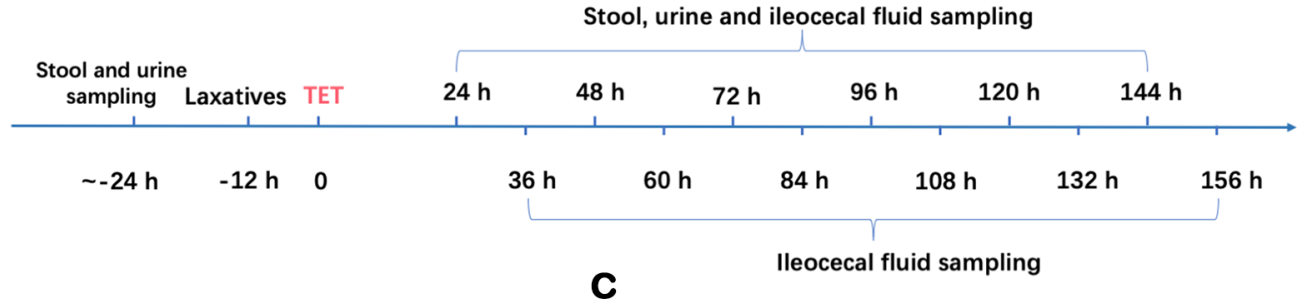

b

d
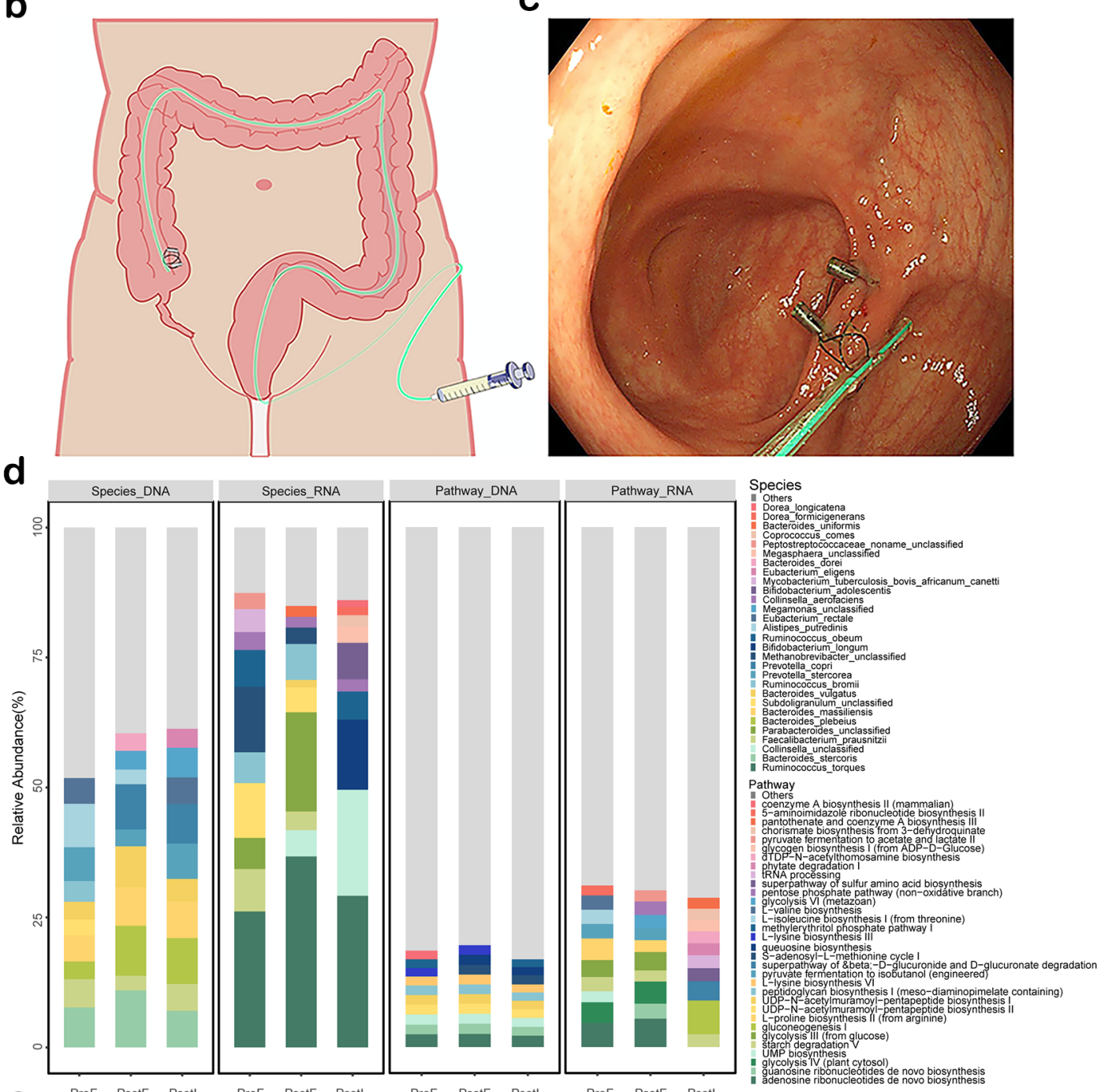

e

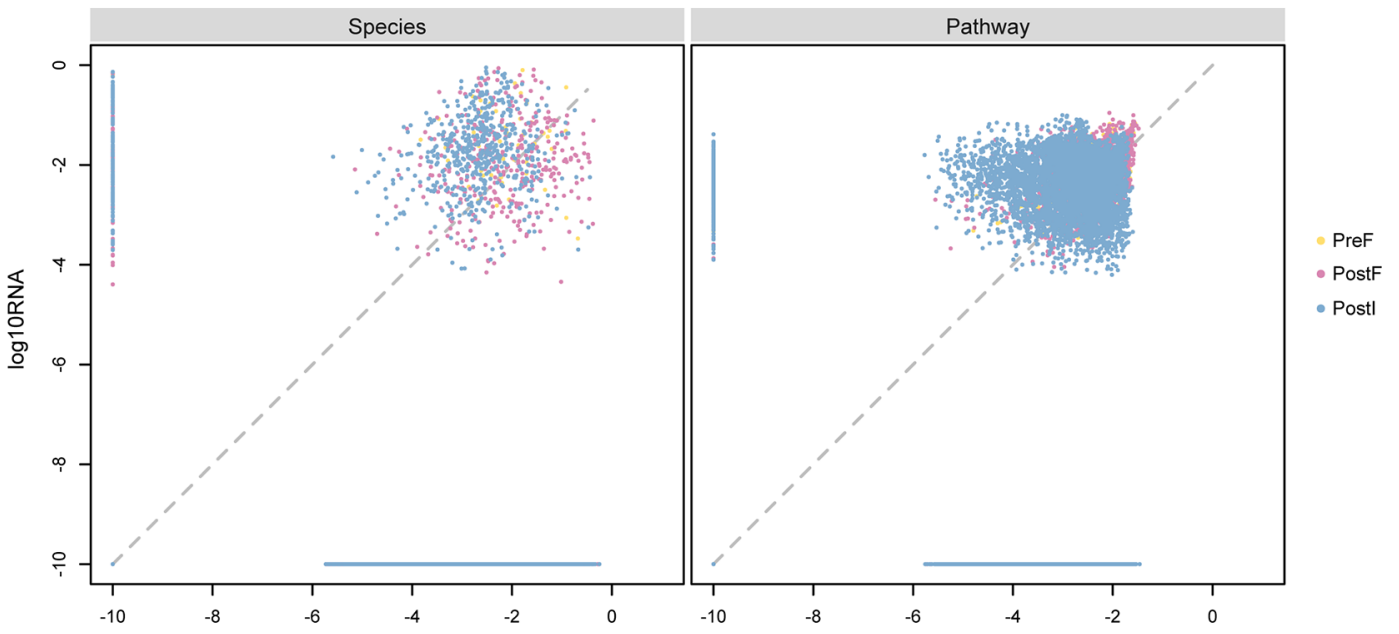


392 Fig. 1 Fecal and ileocecal microbiome are distinct in composition and functionality.

393 (a) Flow chart of sample collection. (b) Schematic diagram of colonic TET; (c)

394 Colonoscopy view of terminal of TET tube fixed on the ileocecal valve by two

395 endoscopic clicks; (d) An overview of relative abundance of composition at species

396 level and functional pathways. Different colors of the bar represent different species or

397 pathways, and the relative abundances are the average of individuals in the three group,

398 fecal samples before osmosis (PreF), fecal samples after osmosis (PostF), and ileocecal

399 samples after osmosis (PostI). Four panels show species composition from metagenome

400 (Species_DNA) and metatranscriptome (Species_RNA), and functional pathways from

401 (Pathway_DNA) and metatranscriptome (Pathway_RNA). Contributions of species

402 and pathway were scaled to sum to 1 respectively within each group; (e) Comparison

403 of metagenome- vs. metatranscriptome-derived species composition and functionality.

$404 \mathrm{X}$ and $\mathrm{Y}$ show $\log (10)$ relative abundance of species and pathways in metagenome

405 (DNA) and metatranscriptome (RNA), added a very small number(10^-10) before to

406 aovid negative infitite values. 
a b

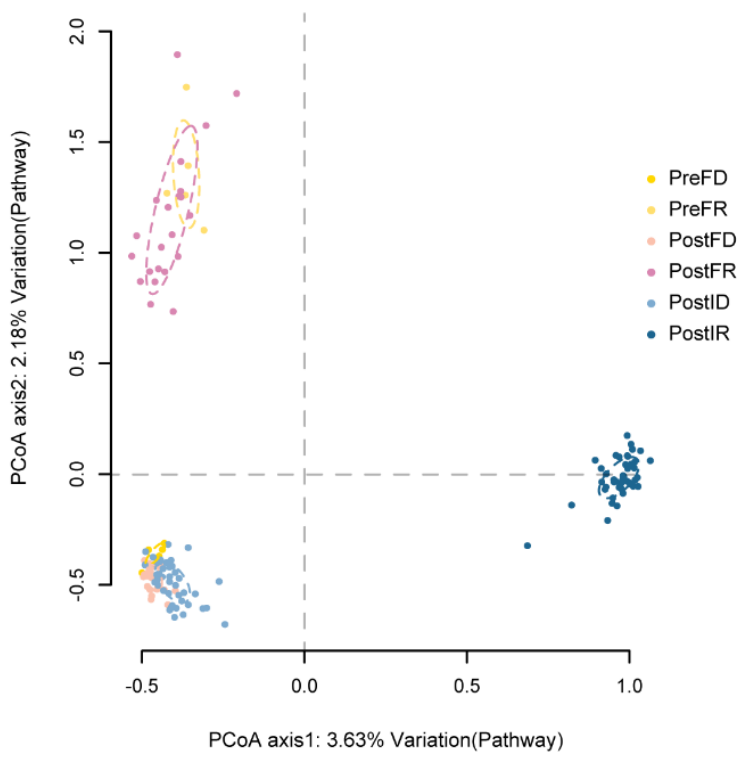

Fig. 2 Dissimilarity between defecated feces and ileocecal samples in species

composition and functional pathways. (a) The principal coordinate analysis (PCoA) of metagenomic (DNA) and metatranscriptomic (RNA) composition at species level for samples grouped by the sampling time and location (PreF, PostF, PostI), with ellipses showing 95\% CI. (b) The principal coordinate analysis (PCoA) of metagenomic (DNA) and metatranscriptomic (RNA) functional pathways for samples grouped by the sampling time and location (PreF, PostF, PostI), with ellipses showing 95\% CI. 
a

415
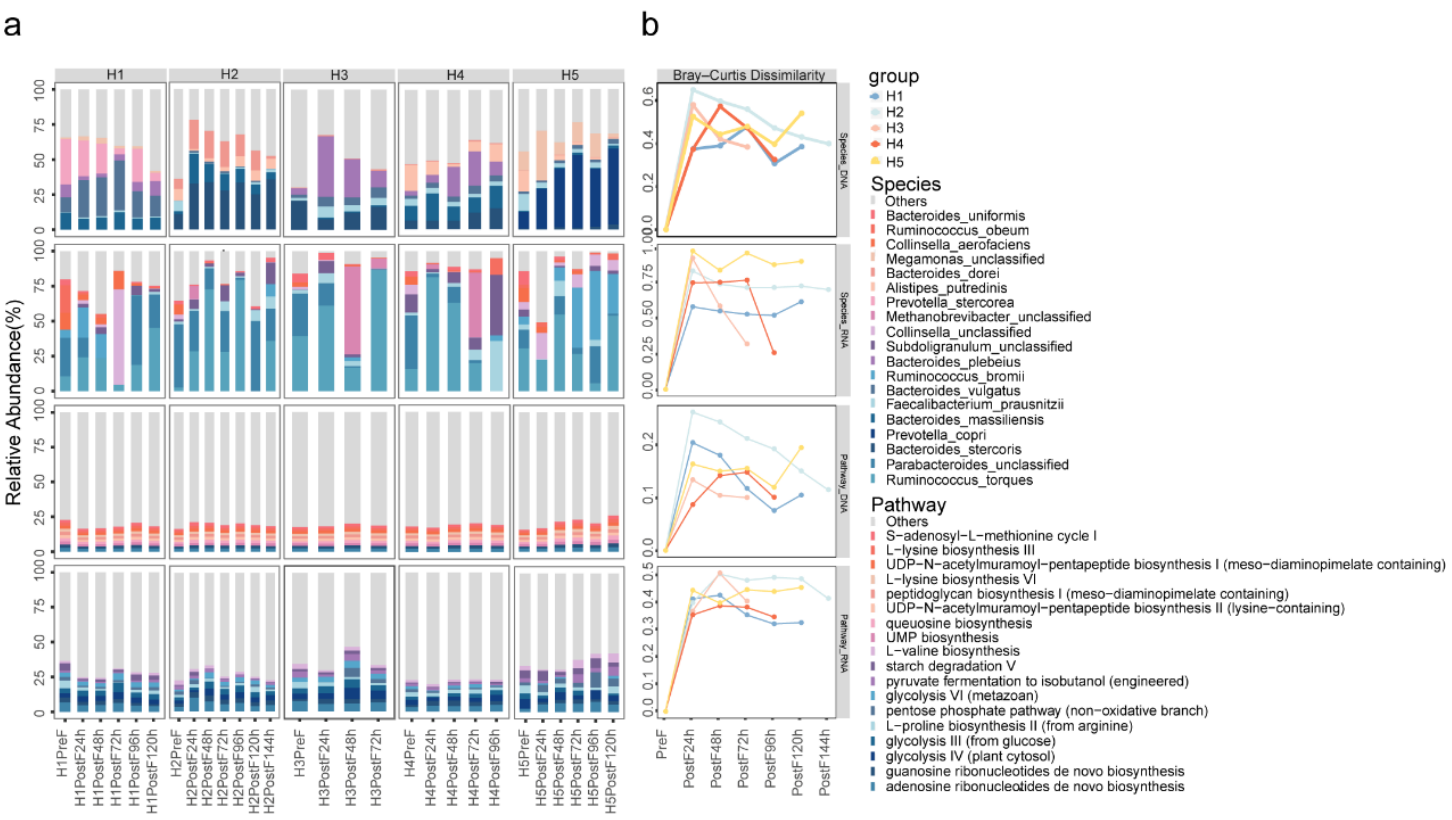

Fig. 3 Dynamic reconstruction after osmotic laxatives of composition and function

in fecal samples. (a) Bar plots show the ten most major species and pathways with the highest relative abundances in each individual $(\mathrm{H} 1, \mathrm{H} 2, \mathrm{H} 3, \mathrm{H} 4, \mathrm{H} 5)$ at each sampling point, including before osmosis (Pre), and every day after osmosis (Post). (b) the BrayCurtis dissimilarities from the fecal samples after osmotic laxatives (Post) to the fecal samples before interference (Pre) in each individual.

a

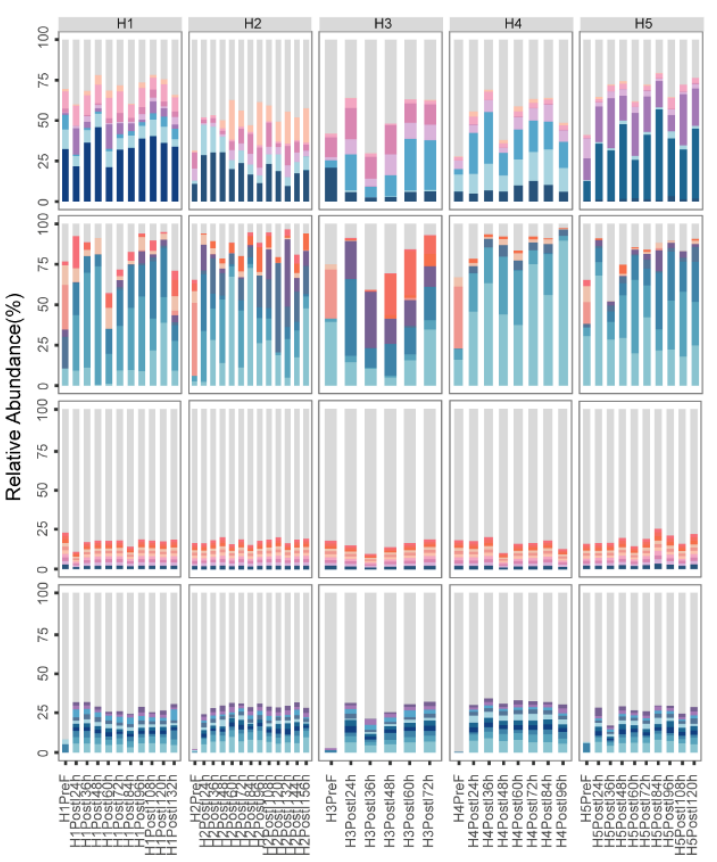

b

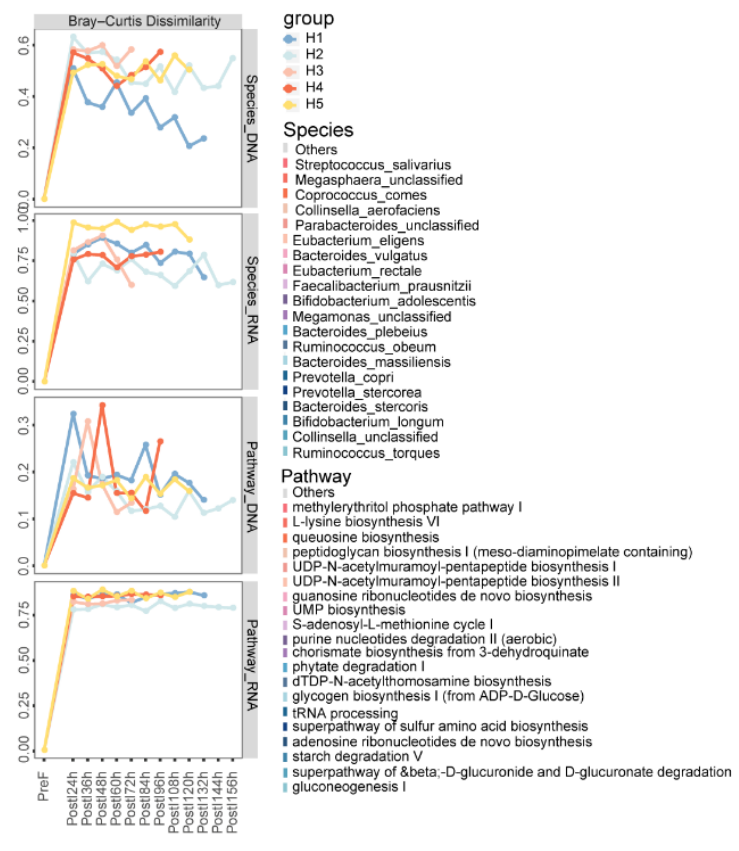



in each individual.
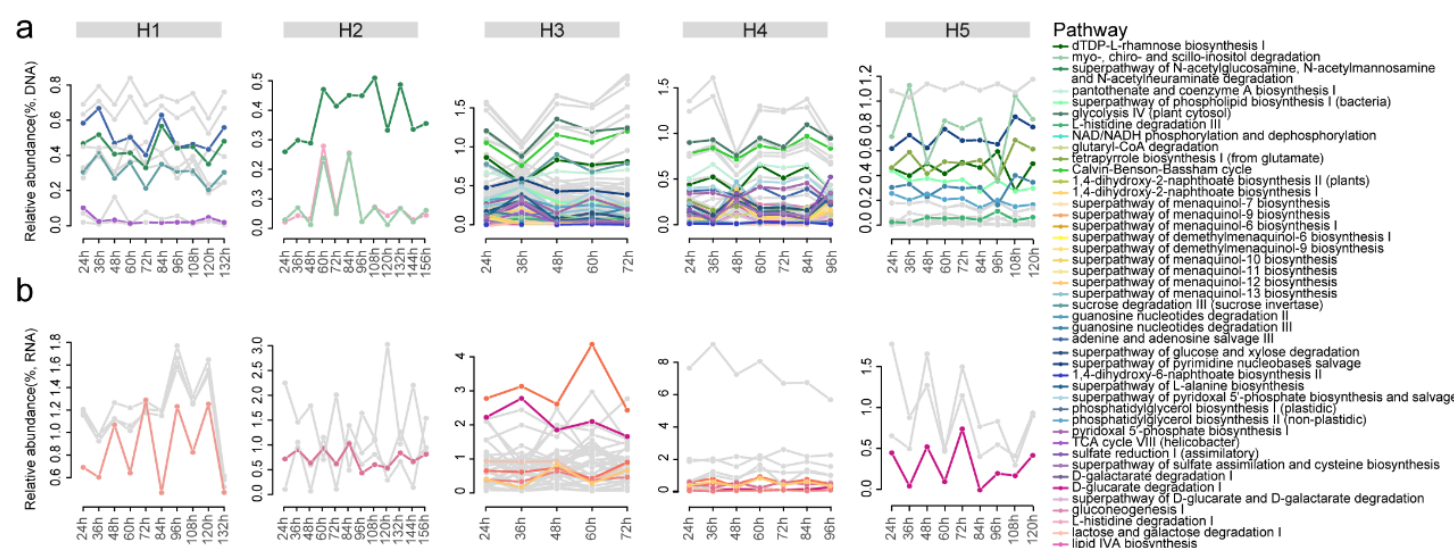

C


Fig. 5 Circadian rhythm of functional pathways and bacterial species of gut

microbiome in ileocecal samples. Metabolic pathways (a for metagenomic data, $b$ for

433 metatranscriptome data) and species (c for metagenomic data, $\mathrm{d}$ for metatranscriptome

434 data) with diurnal oscillations were selected by monitoring changes in their relative

435 abundance. Moreover, these pathways shared by two or more individuals were colored, 
436 and the pathways with diurnal oscillations shown in only one individual were displayed

437 in grey. In contrast, all species with diurnal oscillations due to the small number of 438 species. samples before interference (Pre) in each individual. a

b
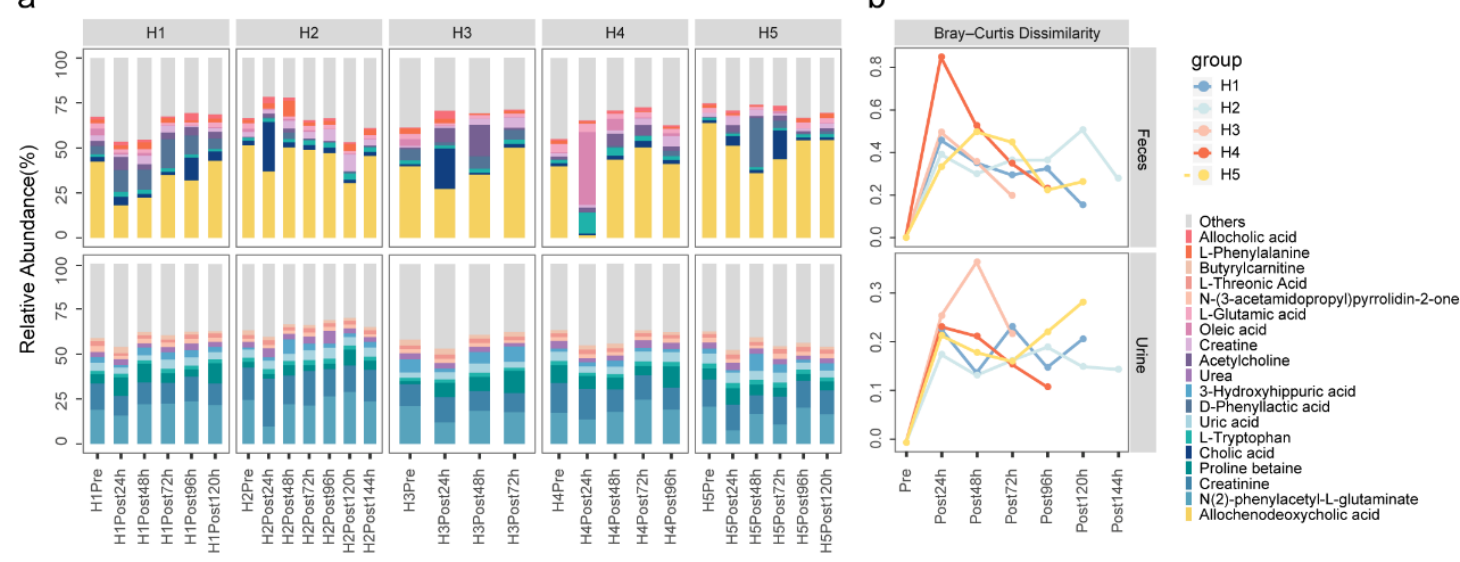

Fig. 6 Dynamic reconstruction after osmotic laxatives of metabolites in fecal and

urinary samples. (a) Bar plots show the major ten metabolites with the highest relative abundances in each individual $(\mathrm{H} 1, \mathrm{H} 2, \mathrm{H} 3, \mathrm{H} 4, \mathrm{H} 5)$ at each sampling time, including fecal and urinary samples collected before (Pre) and after (Post) osmosis; (b) the BrayCurtis dissimilarities from the fecal or urinary samples after osmotic laxatives (Post) to 


\section{Refrences:}

448 1. Lynch, J.B. \& Hsiao, E.Y. Microbiomes as sources of emergent host phenotypes. Science 365, 1405-1409 (2019).

2. Donaldson, G.P., Lee, S.M. \& Mazmanian, S.K. Gut biogeography of the bacterial microbiota. Nat Rev Microbio/ 14, 20-32 (2016).

3. Ahern, P.P. \& Maloy, K.J. Understanding immune-microbiota interactions in the intestine. Immunology 159, 4-14 (2020).

4. Manuzak, J.A. et al. Antibiotic-induced microbiome perturbations are associated with significant alterations to colonic mucosal immunity in rhesus macaques. Mucosal Immuno/

5. Tropini, C. et al. Transient Osmotic Perturbation Causes Long-Term Alteration to the Gut Microbiota. Cel/ 173, 1742-1754 e1717 (2018).

6. Wang, Y. et al. The intestinal microbiota regulates body composition through NFIL3 and the circadian clock. Science 357, 912-916 (2017).

7. Kuang, Z. et al. The intestinal microbiota programs diurnal rhythms in host metabolism

8. Gasparrini, A.J. et al. Antibiotic perturbation of the preterm infant gut microbiome and

9. David, L.A. et al. Diet rapidly and reproducibly alters the human gut microbiome. Nature

10. Johnson, A.J. et al. Daily Sampling Reveals Personalized Diet-Microbiome Associations in

11. Wu, G.D. et al. Linking long-term dietary patterns with gut microbial enterotypes. Science 334, 105-108 (2011).

12. Zimmermann, M., Zimmermann-Kogadeeva, M., Wegmann, R. \& Goodman, A.L. Mapping human microbiome drug metabolism by gut bacteria and their genes. Nature $570,462-$ 467 (2019).

13. Maier, L. et al. Extensive impact of non-antibiotic drugs on human gut bacteria. Nature 
on the gut microbiota in healthy humans. J Antimicrob Chemother 74, 782-786 (2019).

15. Zhernakova, A. et al. Population-based metagenomics analysis reveals markers for gut microbiome composition and diversity. Science 352, 565-569 (2016).

16. Falony, G. et al. Population-level analysis of gut microbiome variation. Science 352, 560564 (2016).

17. Becattini, S., Taur, Y. \& Pamer, E.G. Antibiotic-Induced Changes in the Intestinal Microbiota and Disease. Trends Mol Med 22, 458-478 (2016).

18. Li, S.S. et al. Durable coexistence of donor and recipient strains after fecal microbiota transplantation. Science 352, 586-589 (2016).

19. Bharucha, A.E. \& Lacy, B.E. Mechanisms, Evaluation, and Management of Chronic Constipation. Gastroenterology (2020).

20. Luthra, P. et al. Efficacy of drugs in chronic idiopathic constipation: a systematic review and network meta-analysis. Lancet Gastroenterol Hepato/4, 831-844 (2019).

21. Lozupone, C.A., Stombaugh, J.I., Gordon, J.I., Jansson, J.K. \& Knight, R. Diversity, stability and resilience of the human gut microbiota. Nature 489, 220-230 (2012).

22. Relman, D.A. The human microbiome: ecosystem resilience and health. Nutr Rev 70 Suppl 1, S2-9 (2012).

23. Peng, Z. et al. Colonic transendoscopic enteral tubing: A novel way of transplanting fecal microbiota. Endosc Int Open 4, E610-613 (2016).

24. Ding, X. et al. Long-Term Safety and Efficacy of Fecal Microbiota Transplant in Active Ulcerative Colitis. Drug Saf 42, 869-880 (2019).

25. Mehta, R.S. et al. Stability of the human faecal microbiome in a cohort of adult men. Nat Microbio/3, 347-355 (2018).

26. Schirmer, M. et al. Dynamics of metatranscription in the inflammatory bowel disease gut microbiome. Nat Microbio/3, 337-346 (2018).

27. Chong, J., Wishart, D.S. \& Xia, J. Using MetaboAnalyst 4.0 for Comprehensive and Integrative Metabolomics Data Analysis. Curr Protoc Bioinformatics 68, e86 (2019).

28. Jin, M. et al. Faecal microbiota from patients with cirrhosis has a low capacity to ferment non-digestible carbohydrates into short-chain fatty acids. Liver Int 39, 1437-1447 (2019). 
534 29. Vital, M. et al. Metagenomic Insights into the Degradation of Resistant Starch by Human Gut Microbiota. App/ Environ Microbio/ 84 (2018).

30. Takahashi, K. et al. Reduced Abundance of Butyrate-Producing Bacteria Species in the Fecal Microbial Community in Crohn's Disease. Digestion 93, 59-65 (2016).

31. Rosario, D. et al. Understanding the Representative Gut Microbiota Dysbiosis in Metformin-Treated Type 2 Diabetes Patients Using Genome-Scale Metabolic Modeling. Front Physio/ 9, 775 (2018).

32. Liu, H. et al. Resilience of human gut microbial communities for the long stay with multiple dietary shifts. Gut 68, 2254-2255 (2019).

33. Ng, K.M. et al. Recovery of the Gut Microbiota after Antibiotics Depends on Host Diet, (2019).

34. Liu, C. et al. Alterations and structural resilience of the gut microbiota under dietary fat

35. MacPherson, C.W. et al. Gut Bacterial Microbiota and its Resistome Rapidly Recover to

36. Goethel, A. et al. Nod2 influences microbial resilience and susceptibility to colitis following

37. Mortensen, M.S. et al. Stability and resilience of the intestinal microbiota in children in

38. Louis, P. \& Flint, H.J. Diversity, metabolism and microbial ecology of butyrate-producing

39. Tahara, Y. et al. Gut Microbiota-Derived Short Chain Fatty Acids Induce Circadian Clock

40. Segers, A. et al. The circadian clock regulates the diurnal levels of microbial short-chain fatty acids and their rhythmic effects on colon contractility in mice. Acta Physiol (Oxf) 225,

41. Fellows, R. et al. Microbiota derived short chain fatty acids promote histone crotonylation 
600

601

602

603

604

605

606

607

608

histone-deacetylase activity by short-chain fatty acids and some polyphenol metabolites formed in the colon. J Nutr Biochem 19, 587-593 (2008).

43. Bolger, A.M., Lohse, M. \& Usadel, B. Trimmomatic: a flexible trimmer for Illumina sequence data. Bioinformatics 30, 2114-2120 (2014).

44. Franzosa, E.A. et al. Species-level functional profiling of metagenomes and metatranscriptomes. Nat Methods 15, 962-968 (2018).

45. Segata, N. et al. Metagenomic microbial community profiling using unique clade-specific marker genes. Nature Methods 9, 811-814 (2012).

46. Langmead, B. \& Salzberg, S.L. Fast gapped-read alignment with Bowtie 2. Nature Methods 9, 357-359 (2012).

47. Dunn, W.B. et al. Procedures for large-scale metabolic profiling of serum and plasma using gas chromatography and liquid chromatography coupled to mass spectrometry. Nature Protocols 6, 1060-1083 (2011).

48. Hou, W. et al. A strategy for the targeted metabolomics analysis of 11 gut microbiotahost co-metabolites in rat serum, urine and feces by ultra high performance liquid chromatography-tandem mass spectrometry. J Chromatogr A 1429, 207-217 (2016).

49. Rohart, F., Gautier, B., Singh, A. \& Lê Cao, K.-A. mixOmics: An R package for 'omics feature selection and multiple data integration. PLOS Computational Biology 13, e1005752 (2017).

50. Shannon, P. et al. Cytoscape: a software environment for integrated models of biomolecular interaction networks. Genome Res 13, 2498-2504 (2003). 